\title{
Dipolar Bose gas with three-body interactions in weak disorder
}

\author{
Redaouia Keltoum ${ }^{1}$ and Abdelâali Boudjemâa $a^{1,2}$ \\ 1 Laboratory of Mechanics and Energy, \\ 2 Department of Physics, \\ Faculty of Exact Sciences and Informatics, \\ Hassiba Benbouali University of Chlef, \\ P.O. Box 78, 02000, Chlef, Algeria.
}

\begin{abstract}
We study effects of weak disorder with Gaussian correlation function on a dipolar Bose gas with three-body interactions using the Hartree-Fock-Bogoliubov theory. Corrections due to quantum, thermal and disorder fluctuations to the condensate depletion, the one-body density correlation function as well as to the equation of state and the ground state energy are properly calculated. We show that the intriguing interplay of the disorder, dipole-dipole interactions and three-body interactions plays a fundamental role in the physics of the system. Interestingly, we find that the three-body interactions release atoms localized in the respective minima of the random potential.

PACS numbers: 03.75.Hh, 67.85.De
\end{abstract}

\section{INTRODUCTION}

Since the first observation of a Bose-Einstein condensate (BEC) with dipole-dipole interactions (DDI) in 2005, quantum dilute atomic gases have attracted major attention both theoretically and experimentally [1 4] $]$. Dipolar BECs provide rich new physics, not encountered in systems with the contact interaction, thanks to the longrange and anisotropic character of the DDI.

The properties of dipolar BECs in weak random potential have recently sparked a great interest [5-11]. It is widely agreed that the stability and the shape of such systems depend critically on the interplay between the DDI and the disorder potential. For instance, the superfluidity acquires a characteristic direction dependence due to the anisotropy of the DDI [5 $[9]$. The studies of two-dimensional (2D) disordered dipolar gases may offer the possibility for the observation of the superglass state [10, 11]. Most recently, impacts of the Lee-Huang-Yang (LHY) quantum corrections on a dirty dipolar Bose gas have been analyzed by one of us [12] using a perturbative theory. It is found that the LHY quantum fluctuations lead to reduce the disorder effects inside the condensate preventing the formation of the Bose glass state.

On the other hand, three-body interactions (TBI) play a key role in a wide variety of interesting physical phenomena, and provide a new physics not existed in systems with two-body interactions. Inelastic three-body processes, including observations of Efimov quantum states and atom loss from recombination have been reported in Refs [13 17]. Weakly interacting Bose and Fermi gases with competing attractive two-body and large repulsive TBI may form droplets [18]. Effects of TBI in ultracold bosonic atoms loaded in an optical lattice or a superlattice were also studied in [19 22]. It was shown also that the TBI in Bose condensate may singnificantly modify the collective excitations [23 25], the transition temperature, the condensate depletion and the stability of a BEC [26, 27]. In the context of ultracold atoms with DDI, it has been revealed that the combined effects of
TBI and DDI may lead to the formation of a stable supersolid state 28] and a quantum droplet state 29 33. Very recently, we have shown that the TBI may shift the density profiles, the condensed fraction and the collective modes of a dipolar condensate at finite temperature [34].

Disordered dipolar Bose gases with TBI present a different physical picture and may open prospects to achieve a stable superfluid. The goal of this work is then to study, for the first time to the best of our knowledge, effects of a weak disorder potential with Gaussian correlation function on the properties of $\mathrm{BEC}$ with two-body interactions and TBI. To this end, we use the Hatree-Fock-Bogoliubov (HFB) theory which includes an additional TBI term in the momentum space. Our results show that the TBI are relevant in reducing the influence of the disorder potential in BEC. Impacts of the disorder potential and the TBI on the fluctuations, coherence and the thermodynamics of the condensate are also highlighted. We compare our findings with previous theoretical results.

The rest of the paper is organized as follows. Section introduces the HFB model for a disordered dipolar Bose gases with two-body interactions and TBI. In Sec WII we use a correlated Gaussian disorder potential to illustrate our model and derive useful expressions for the disorder fraction and the noncondensed density. In Sec IV we look at how the interplay of the TBI and the disorder potential enhance the coherence of the system by numerically analyzing the behavior of the one-body density matrix. In Sec $\square]$ we calculate corrections due to the disorder effects and TBI to the chemical potential and the ground state energy. Our conclusions are drawn in SecVI.

\section{MODEL}

We consider the effects of an external random potential $U(\mathbf{r})$ on a dilute $3 \mathrm{D}$ dipolar Bose gas with contact twoand three-body interactions. Assuming that dipoles are oriented along $z$-axis. The Hamiltonian of the system 
reads:

$$
\begin{aligned}
\hat{H} & =\int d \mathbf{r} \hat{\psi}^{\dagger}(\mathbf{r})\left[-\frac{\hbar^{2}}{2 m} \Delta+U(\mathbf{r})\right] \hat{\psi}(\mathbf{r}) \\
& +\frac{1}{2} \int d \mathbf{r} \int d \mathbf{r}^{\prime} \hat{\psi}^{\dagger}(\mathbf{r}) \hat{\psi}^{\dagger}\left(\mathbf{r}^{\prime}\right) V\left(\mathbf{r}-\mathbf{r}^{\prime}\right) \hat{\psi}\left(\mathbf{r}^{\prime}\right) \hat{\psi}(\mathbf{r}) \\
& +\frac{g_{3}}{6} \int d \mathbf{r} \hat{\psi}^{\dagger}(\mathbf{r}) \hat{\psi}^{\dagger}(\mathbf{r}) \hat{\psi}^{\dagger}(\mathbf{r}) \hat{\psi}(\mathbf{r}) \hat{\psi}(\mathbf{r}) \hat{\psi}(\mathbf{r})
\end{aligned}
$$

where $\hat{\psi}^{\dagger}$ and $\hat{\psi}$ denote, respectiveiy the usual creation and annihilation field operators, $m$ is the particle mass. The two-body interactions is described by the potential $V\left(\mathbf{r}-\mathbf{r}^{\prime}\right)=g_{2} \delta\left(\mathbf{r}-\mathbf{r}^{\prime}\right)+V_{d d}\left(\mathbf{r}-\mathbf{r}^{\prime}\right)$, where $g_{2}=4 \pi \hbar^{2} a / m$ with $a$ being the $s$-wave scattering length is assumed to be positive. The DDI term $V_{d d}(\mathbf{r})=\mathcal{M}_{0} \mathcal{M}^{2}\left(1-3 \cos ^{2} \theta\right) / 4 \pi r^{3}$, where $\mathcal{M}$ is the magnetic moment and $\theta$ is the angle between the relative position of particles $\mathbf{r}$ and $z$-axis. The three-body coupling constant $g_{3}$ is in general a complex number with $\operatorname{Im}\left(g_{3}\right)$ describing the three-body recombination loss and $\operatorname{Re}\left(g_{3}\right)$ quantifying the three-body scattering parameter. Here, we will assume that the imaginary part of $g_{3}$ is negligible [15, 24, 31, 35, 36] which means that the loss rate is sufficiently small and hence, the system is stable. This well coincides with the experimental conditions reported in Ref.[29]. Note that the strength of the three-body coupling $g_{3}$ is related to the atomic species and can be adjusted by Feshbach resonance [30, 37]. It is therefore, hard to predict the exact value of $g_{3}$ (see e.g. [18, 35, 38]).

In what follows, we suppose that the disorder potential is described by vanishing ensemble averages $\langle U(\mathbf{r})\rangle=0$ and a finite disorder correlation function $\left\langle U(\mathbf{r}) U\left(\mathbf{r}^{\prime}\right)\right\rangle=$ $R\left(\mathbf{r}, \mathbf{r}^{\prime}\right)$.

In the frame of the HFB formalism, the Bose-field operator can be written as

$$
\hat{\psi}(\mathbf{r}, t)=\Phi(\mathbf{r}, t)+\hat{\bar{\psi}}(\mathbf{r}, t),
$$

where $\Phi$ is the condensate wavefunction, and $\hat{\bar{\psi}}$ stands for the field of the noncondensed thermal atoms. Working in Fourier space, the condensate wavefunction is taken as $\Phi(\mathbf{r}, t)=\sqrt{n_{c}}$ with $n_{c}$ being the condensate density, and the field operator of noncondensed atoms can be expanded in terms of plane waves $\hat{\bar{\psi}}=(1 / \sqrt{V}) \sum_{k} a_{k} e^{i \mathbf{k} . \mathbf{r}}$. The DDI potential in momentum space is given by: $V_{d d}(\mathbf{k})=\left(\mu_{0} \mu^{2} / 12 \pi\right)\left(3 \cos ^{2} \theta_{\mathbf{k}}-1\right)$, where the vector $\mathbf{k}$ represents the momentum transfer imparted by the collision.

Now we deal with a weakly interacting system and assume that the disorder is sufficiently weak. Then it is possible to use the Bogoliubov-Huang-Meng approach [39] which suggests the transformation:

$$
\hat{a}_{k}=u_{k} \hat{b}_{k}-v_{k} \hat{b}_{-k}^{\dagger}-\beta_{k}, \quad \hat{a}_{k}^{\dagger}=u_{k} \hat{b}_{k}^{\dagger}-v_{k} \hat{b}_{-k}-\beta_{k}^{*},
$$

where $\hat{b}_{k}^{\dagger}$ and $\hat{b}_{k}$ are operators of elementary excitations, the functions $u_{k}, v_{k}$ are defined as $u_{k}, v_{k}=\left(\sqrt{\varepsilon_{k} / E_{k}} \pm\right.$
$\left.\sqrt{E_{k} / \varepsilon_{k}}\right) / 2$ with $E_{k}=\hbar^{2} k^{2} / 2 m$ being the free particle energy, and

$$
\beta_{k}=\sqrt{\frac{n_{c}}{V}} \frac{E_{k}}{\varepsilon_{k}^{2}} U_{k}
$$

The Bogoliubov excitations energy is given by

$$
\varepsilon_{k}=\sqrt{E_{k}^{2}+2 n_{c} E_{k} \bar{V}(\mathbf{k})},
$$

where $\bar{V}(\mathbf{k})=g_{2}\left(1+g_{3} n_{c} / g_{2}\right)\left[1+\gamma\left(3 \cos ^{2} \theta_{\mathbf{k}}-1\right)\right]$ with $\gamma=\epsilon_{d d} /\left(1+g_{3} n_{c} / g_{2}\right), \epsilon_{d d}=\mathcal{M}_{0} \mathcal{M}^{2} /\left(3 g_{2}\right)$ is the relative strength between the DDI and short-range interactions. For $k \rightarrow 0$, the excitations are sound waves $\varepsilon_{k}=\hbar c_{s}\left(\theta_{\mathbf{k}}\right) k$, where $c_{s}\left(\theta_{\mathbf{k}}\right)=c_{0} \sqrt{\left(1+g_{3} n_{c} / g_{2}\right)\left[1+\gamma\left(3 \cos ^{2} \theta_{\mathbf{k}}-1\right)\right]}$ with $c_{0}=\sqrt{g_{2} n_{c} / m}$ being the sound velocity without DDI and TBI.

The diagonal form of the Hamiltonian (11) can be written as $\hat{H}=E+\sum_{k} \varepsilon_{k} \hat{b}_{k}^{\dagger} \hat{b}_{k}$. The total energy $E=$ $E_{0}(\theta)+\delta E+E_{R}$, where the zeroth order term

$$
E_{0}(\theta)=\bar{V}(\theta) n_{c} N_{c} / 2,
$$

which should be computed in the limit $k \rightarrow 0$ since it accounts for the condensate (lowest state). The groundstate energy shift due to quantum fluctuations is

$$
\delta E=\frac{1}{2} \sum_{k}\left[\varepsilon_{k}-E_{k}-n_{c} \bar{V}(\mathbf{k})\right],
$$

and

$$
E_{R}=-\sum_{k} n_{c}\left\langle\left|U_{k}\right|^{2}\right\rangle \frac{E_{k}}{\varepsilon_{k}^{2}}=-\sum_{k} n_{c} R_{k} \frac{E_{k}}{\varepsilon_{k}^{2}},
$$

gives the correction to the ground-state energy due to the external random potential.

The noncondensed density is defined as

$$
\tilde{n}=\sum_{k}\left\langle\hat{a}_{k}^{\dagger} \hat{a}_{k}\right\rangle=\frac{1}{V} \sum_{k}\left[\left(u_{k}^{2}+v_{k}^{2}\right) N_{k}+v_{k}^{2}+\left\langle\left|\beta_{\mathbf{k}}\right|^{2}\right\rangle\right],
$$

where $N_{k}=\left\langle\hat{b}_{k}^{\dagger} \hat{b}_{k}\right\rangle=1 /\left[\exp \left(\varepsilon_{k} / T\right)-1\right]$ is the BoseEinstein distribution function, and the rest of the expectation values equal to zero $\left(\left\langle\hat{b}_{k}^{\dagger} \hat{b}_{k}^{\dagger}\right\rangle=\left\langle\hat{b}_{k} \hat{b}_{k}\right\rangle=0\right)$.

Inserting the expressions of $u_{k}$ and $v_{k}$ in Eq.(9), and working in the thermodynamic limit where the sum over $k$ can be replaced by the integral $\sum_{\mathbf{k}}=V \int d \mathbf{k} /(2 \pi)^{3}$, we get

$$
\begin{aligned}
\tilde{n} & =\tilde{n}_{0}+\tilde{n}_{t h}+n_{R} \\
& =\frac{1}{2} \int \frac{d \mathbf{k}}{(2 \pi)^{3}}\left[\frac{E_{k}+\bar{V}(\mathbf{k}) n_{c}}{\varepsilon_{k}}-1\right] \\
& +\frac{1}{2} \int \frac{d \mathbf{k}}{(2 \pi)^{3}} \frac{E_{k}+\bar{V}(\mathbf{k}) n_{c}}{\varepsilon_{k}}\left[\operatorname{coth}\left(\frac{\varepsilon_{k}}{2 T}\right)-1\right] \\
& +n_{c} \int \frac{d \mathbf{k}}{(2 \pi)^{3}} R_{k} \frac{E_{k}^{2}}{\varepsilon_{k}^{4}}
\end{aligned}
$$


The leading term (10b) denotes the zero temperature contribution to the noncondensed density. The subleading term (10c) stands for thermal fluctuation corrections to the noncondensed density. Whereas the third term (10d) represents the condensate fluctuations due to the disorder potential known as glassy fraction and originates from the accumulation of density near the potential minima and density depletion around the maxima.

\section{GAUSSIAN-CORRELATED DISORDER}

As a concrete example, we consider in this section the case of a correlated Gaussian disorder model, which allow for unique control of the interplay between the disorder potential and interactions in both dipolar and nondipolar BEC [5, 9]. It can be written as

$$
R(k)=R_{0} \exp \left[-\sigma^{2} k^{2} / 2\right],
$$

where $R_{0}$ is the disorder strength which has dimension (energy $)^{2} \times(\text { length })^{3}$ and $\sigma$ characterizes the correlation length of the disorder.

The glassy fraction can be calculated easily via Eq.(10d)

$$
n_{R}=n_{\mathrm{HM}}\left(1+g_{3} n_{c} / g_{2}\right)^{-1 / 2} h(\gamma, \sigma / \xi),
$$

where $n_{\mathrm{HM}}=\left[m^{2} R_{0} / 8 \pi^{3 / 2} \hbar^{4}\right] \sqrt{n_{c} / a}$ is the usual HuangMeng result [39]. The anisotropic disorder function is given as

$$
h(\gamma, \sigma / \xi)=\int_{0}^{\pi} \mathrm{d} \theta \frac{\sin \theta S(\alpha)}{2 \sqrt{1+\gamma\left(3 \cos ^{2} \theta-1\right)}},
$$

where the function $S(\alpha)=e^{2 \alpha}(4 \alpha+1)[1-\operatorname{erf}(\sqrt{2 \alpha})]-$ $2 \sqrt{2 \alpha / \pi}$, and $\alpha=\sigma^{2}\left[\epsilon_{d d} / \gamma\left(1+\gamma\left(3 \cos ^{2} \theta-1\right)\right)\right] / \xi^{2}$ with $\xi=\hbar / \sqrt{m n_{c} g_{2}}$ being the healing length. In the absence of the DDI $\left(\epsilon_{d d}=0\right)$, and in the limit $\sigma / \xi \rightarrow 0$ and $g_{3}=0$, one has $h(\gamma, \alpha) \rightarrow 1$, thus, one recovers the wellknown Hang and Meng result $\left(n_{R}=n_{\mathrm{HM}}\right)$ 39.

The effects of both correlation length and effective interaction parameter $\gamma$ on the behavior of the disorder function are presented in Fig[1. We observe that the function $h(\gamma, \alpha)$ is decreasing with $g_{3} n_{c} / g_{2}$ indicating that the TBI lead to reduce the disorder fluctuations (glassy fraction) inside the condensate even in the limit $\sigma<\xi$. As is expected, the disorder fraction becomes significant for large DDI in contrast to the case of a disordered dipolar BEC with Lee-Huang-Yang (LHY) quantum corrections [12]. The main difference between the TBI and the LHY corrections is that these latter are valid only in the regime of weak disorder since they are computed within the local density approximation which assumes that the external random potential should vary smoothly in space on a length scale comparable to the healing length or the characteristic correlation length of the disorder [12]. Whereas, the TBI still remain applicable for both weak and strong disorder potentials. For $\sigma>\xi$, the disorder effects is not important (see Fig[1,b).
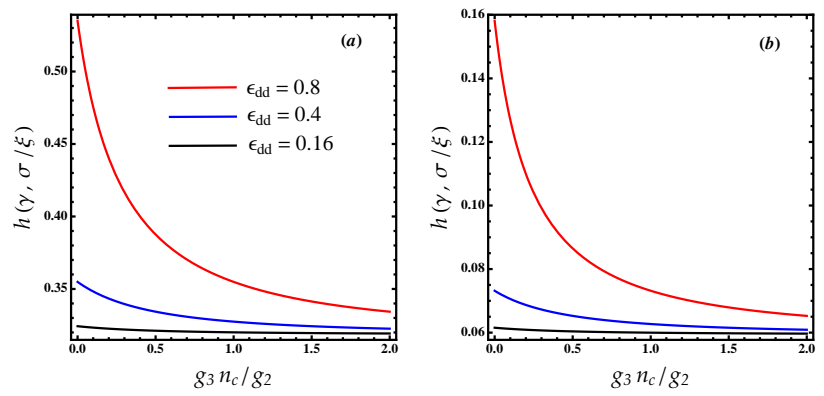

FIG. 1. Disorder function $h(\gamma, \sigma / \xi)$, as a function of $g_{3} n_{c} / g_{2}$ for several values of $\epsilon_{d d}$ for $\sigma / \xi=0.4$ (a) and $\sigma / \xi=1.2$ (b).

For delta-correlated disorder where $\sigma / \xi \rightarrow 0$, the function $h(\gamma, 0)=\mathcal{Q}_{-1}(\gamma)$ and $n_{R}=n_{H M} \mathcal{Q}_{-1}(\gamma)$, where the contribution of the DDI is expressed by the functions $\mathcal{Q}_{j}(x)=\int_{0}^{1} d y\left(1-x+3 x y^{2}\right)^{j / 2}[5,7-9,12]$. Note that the functions $\mathcal{Q}_{j}(x)$ tend to unity for $\gamma=0\left(\mathcal{Q}_{j}(0)=1\right)$, and become imaginary for $\gamma>0$.

Now, we focus ourselves to calculate quantum and thermal depletion in a disordered BEC. Integrals (10b) and (10c) yield, respectively

$$
\frac{\tilde{n}_{0}}{n_{c}}=\frac{8}{3} \sqrt{\frac{n_{c} a^{3}}{\pi}}\left(1+g_{3} n_{c} / g_{2}\right)^{3 / 2} \mathcal{Q}_{3}(\gamma),
$$

and

$$
\frac{\tilde{n}_{t h}}{n_{c}}=\frac{2}{3}\left(\frac{\pi T}{n_{c} g_{2}}\right)^{2} \sqrt{\frac{n_{c} a^{3}}{\pi}}\left(1+g_{3} n_{c} / g_{2}\right)^{-1 / 2} \mathcal{Q}_{-1}(\gamma) .
$$

For $\epsilon_{d d}=0$ and $g_{3}=0$, we recover the standard expressions for $\tilde{n}_{0}$ and $\tilde{n}_{t h}$. When $g_{3}=0$, Eqs. (14) and (15) reduce to that obtained in our previous work for a dipolar BEC without TBI [9].

\section{ONE-BODY DENSITY MATRIX}

The one-body density matrix (first-order correlation function) is defined as $g^{(1)}\left(\mathbf{r}, \mathbf{r}^{\prime}, t, t^{\prime}\right)=\left\langle\hat{\psi}^{\dagger}(\mathbf{r}, t) \hat{\psi}\left(\mathbf{r}^{\prime}, t^{\prime}\right)\right\rangle$. In uniform case it depends only on the difference $\left|\mathbf{r}-\mathbf{r}^{\prime}\right|=r$. Using the decomposition (2), expressing the noncondensed field operator as $\hat{\bar{\psi}}=$ $(1 / V) \sum_{k}\left[u_{k} \hat{b}_{k} e^{i \mathbf{k} \cdot \mathbf{r}}-v_{k} \hat{b}_{k}^{\dagger} e^{-i \mathbf{k} \cdot \mathbf{r}}\right]$, and then taking into account that $|\Phi(\mathbf{r}, t)|=\sqrt{n_{c}}$. We thus, get

$g^{(1)}(\mathbf{r})=n_{c}+g_{R}^{(1)}(\mathbf{r})+\int_{0}^{\infty} \frac{d \mathbf{k}}{(2 \pi)^{d}}\left[v_{k}^{2}+\left(u_{k}^{2}+v_{k}^{2}\right) N_{k}\right] e^{i \mathbf{k} \cdot \mathbf{r}}$,

The second term $g_{R}^{(1)}(\mathbf{r})=\int\left(d \mathbf{k} /(2 \pi)^{3}\right)\left\langle\left|\beta_{\mathbf{k}}\right|^{2}\right\rangle \mathrm{e}^{i \mathbf{k} . \mathbf{r}}$ represents the disorder effects on the first order correlation function. The behavior of $g_{R}^{(1)}(\mathbf{r})$ is displayed in Fig,2, We observe that for small disorder correlation length 

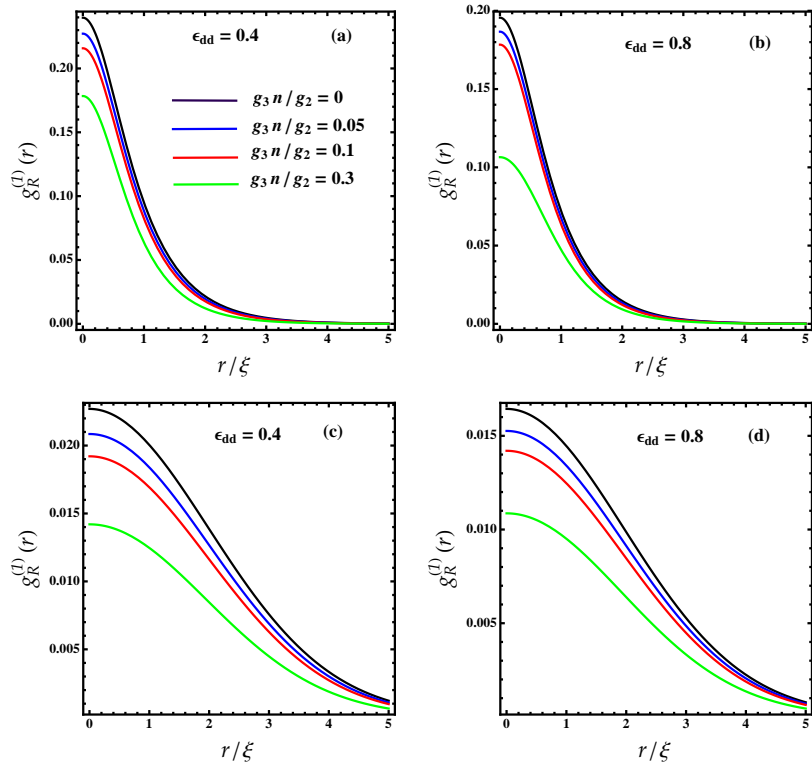

FIG. 2. (color online) One-body density matrix due to the disorder corrections, $\mathrm{g}_{R}^{(1)}(r)$, for $\sigma / \xi=0.2(\mathrm{a})$-(b) and $\sigma / \xi=$ $1.2(\mathrm{c})-(\mathrm{d})$.

$(\sigma / \xi=0.2), g_{R}^{(1)}(r)$ is decreasing with increasing the TBI and the DDI (see Fig 2, a-b ). The same behavior holds for large $\sigma$. Importantly, $g_{R}^{(1)}(r)$ vanishes at large distance $r$ in both cases signaling the non-existence of mini condensates formed by the localized particles in the respective minima of the external random potential. This does not mean that the long-range order of the whole system is destroyed.

The last term in Eq. (16) accounts for the quantum and thermal contributions to the one-body correlation function. One can easily show that this term decays at $r \rightarrow \infty$ and thus, $g^{(1)}(\mathbf{r})$ tends to $n_{c}$, revealing the existence of the long-range order (true condensate). Note that the DDI, the TBI and the temperature can also shift the one-body correlation function.

\section{THERMODYNAMIC QUANTITIES}

In this section, we calculate disorder corrections to some thermodynamic quantities such as the chemical potential and the ground state energy.

Within the realm of the HFB theory, the chemical potential can be written as

$$
\mu=\mu_{0}+\delta \mu+2 \mu_{R}
$$

where

$$
\mu_{0}=\bar{V}(0) n_{c}
$$

is the first-order chemical potential [40].

Corrections to the chemical potential due to the disorder

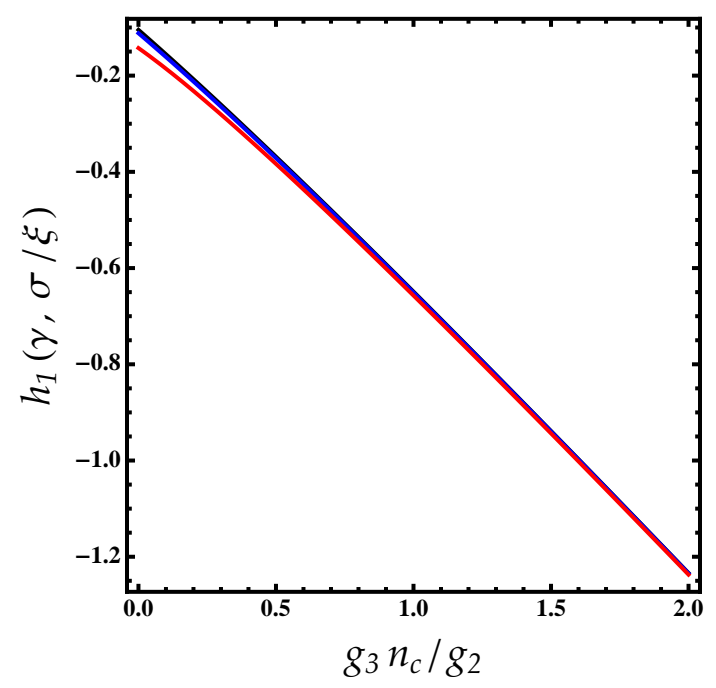

FIG. 3. (Color online) Disorder energy function $h_{1}(\gamma, \sigma / \xi)$ versus $g_{3} n_{c} / g_{2}$ for $\sigma / \xi=0.5$. Black line: $\epsilon_{d d}=0.16$, blue line: $\epsilon_{d d}=0.4$, and red line: $\epsilon_{d d}=0.8$.

effects are given as

$$
\mu_{R}=g_{2} n_{\mathrm{HM}}\left(1+g_{3} n_{c} / g_{2}\right)^{1 / 2} H(\gamma, \sigma / \xi),
$$

where

$$
H(\gamma, \sigma / \xi)=\frac{1}{2} \int_{0}^{\pi} \mathrm{d} \theta \sin \theta \sqrt{1+\gamma\left(3 \cos ^{2} \theta-1\right)} S(\alpha),
$$

Corrections to the chemical potential due to the quantum and thermal fluctuations are defined as : $\delta \mu=$ $\sum_{\mathbf{k}} \bar{V}(\mathbf{k})\left[v_{k}\left(v_{k}-u_{k}\right)+\left(v_{k}-u_{k}\right)^{2} N_{k}\right]$ [33, 40]. Nevertheless, this chemical potential cannot be evaluated straightforwardly since the zero-temperature term is ultraviolet divergent. Such a problem can be worked out either by using the dimensional regularization which is valid for very dilute gases [41 43] or by renormalizing the contact interaction through the $T$-matrix approach [44]. After some algebra, the resulting corrections to the chemical potential read

$$
\begin{aligned}
\frac{\delta \mu}{g_{2} n_{c}} & =\frac{32}{3} \sqrt{\frac{n_{c} a^{3}}{\pi}}\left(1+g_{3} n_{c} / g_{2}\right)^{5 / 2} \mathcal{Q}_{5}(\gamma) \\
& +\frac{2}{3}\left(\frac{\pi T}{n_{c} g_{2}}\right)^{2} \sqrt{\frac{n_{c} a^{3}}{\pi}}\left(1+g_{3} n_{c} / g_{2}\right)^{1 / 2} \mathcal{Q}_{1}(\gamma)
\end{aligned}
$$

Importantly, for $g_{3}=0$, the total chemical potential (17) reduces to that obtained in our recent work [40]. For a cleaned $\left(R_{0}=0\right)$ condensate with two-body contact interactions $\left(g_{3}=\epsilon_{d d}=0\right)$, the obtained corrections to the chemical potential coincide with the seminal Lee-Huang-Yang quantum corrected equation of state [45. 
The energy shift (8) due to the disorder effects is finite and it can be evaluated as

$$
\frac{E_{R}}{N}=\frac{2 m R_{0}}{\hbar^{2}}\left(1+g_{3} n_{c} / g_{2}\right)^{1 / 2} \sqrt{\frac{n_{c} a}{\pi}} \mathcal{H}(\gamma, \sigma / \xi),
$$

where the function

$$
\mathcal{H}(\gamma, \sigma / \xi)=\frac{1}{2} \int_{0}^{\pi} \mathrm{d} \theta \sin \theta \sqrt{1+\gamma\left(3 \cos ^{2} \theta-1\right)} S_{1}(\alpha)
$$

and the function $S_{1}(\alpha)=e^{2 \alpha} \operatorname{erfc}(\sqrt{2 \alpha})-\sqrt{1 / 2 \alpha}$. The disorder energy function $h_{1}(\gamma, \sigma / \xi)$ is decreasing with $g_{3}$ as is seen in Fig 3 indicating that the TBI lead to lower the energy due to the disorder fluctuations which is in agreement with the above results. We observe also that for $g_{3} n_{c} / g_{2} \leq 0.7$, the DDI effects on the energy are more pronounced.

Corrections to the energy due to the quantum and thermal fluctuations can be calculated easily through Eq.(7) or by integrating the chemical potential (21) with respect to the density.

\section{CONCLUSION}

In this paper, we investigated the properties of dipolar Bose gas with TBI subjected to a correlated Gaussian disorder. We showed that the DDI may lead to arrest transport of atoms under disorder augmenting the glassy fraction inside the condensate, while the presence of the TBI may lead to a diffusive motion of particles. We pointed out that the one-body density matrix is a decreasing function with the TBI. We calculated in addition the chemical potential of a disordered dipolar BEC and ultraviolet divergences are removed by means of dimensional regularization. The combined effects of the DDI, TBI, and temperature found to crucially affect the chemical potential and the ground state energy of the system.

\section{AUTHOR CONTRIBUTIONS}

All authors discussed the results and made critical contributions to the work. AB contributed to the writing of the manuscript.
[1] See for review: M. A. Baranov, Physics Reports 464, 71 (2008).

[2] See for review: T. Lahaye et al., Rep. Prog. Phys. 72, 126401 (2009).

[3] See for review: L.D. Carr, D. DeMille, R.V. Krems, and J. Ye, New Journal of Physics 11, 055049 (2009).

[4] See for review: M.A. Baranov, M. Delmonte, G. Pupillo, and P. Zoller, Chemical Reviews, 112, 5012 (2012).

[5] C. Krumnow and A. Pelster, Phys. Rev. A 84, 021608(R) (2011).

[6] B. Nikolic, A. Balaz, and A. Pelster, Phys. Rev. A 88, 013624 (2013).

[7] M. Ghabour and A. Pelster, Phys. Rev. A 90, 063636 (2014).

[8] A. Boudjemâa, Phys. Rev. A 91, 053619 (2015).

[9] A. Boudjemâa, Low Temp. Phys. 180, 377 (2015).

[10] A. Boudjemâa, Phys. Lett. A 379, 2484 (2015).

[11] A. Boudjemâa, J. Phys. B: At. Mol. Opt. Phys. 49, 105301 (2016).

[12] A. Boudjemâa, arXiv:1707.07477 (2017).

[13] V. Efimov Phys. Lett. B 33, 563 (1970).

[14] V. Efimov, Sov. J. Nucl. Phys. 12589 (1971).

[15] P F Bedaque, E. Braaten and H-W-Hammer, Phys. Rev. Lett. 85908 (2000).

[16] T. Kraemer et al. Nature 440, 315 (2006).

[17] E. A. Burt, R. W. Ghrist, C. J. Myatt, M J. Holland, E. A. Cornell and C.E. Wieman, Phys. Rev. Lett. 79337 (1997).

[18] A. Bulgac, Phys. Rev. Lett. 89, 050402 (2002).

[19] A. J. Daley, J.M. Taylor, S. Diehl, M. Baranov, and P. Zoller, Phys. Rev. Lett. 102, 040402 (2009).

[20] L. Mazza, M. Rizzi, M. Lewenstein, and J. I. Cirac, Phys. Rev. A 82, 043629 (2010).
[21] M. Singh, A. Dhar, T. Mishra, R. V. Pai, B. P. Das, Phys. Rev. A 85, 051604 (2012).

[22] K.W. Mahmud and E. Tiesinga, Phys. Rev. A 88, 023602 (2013).

[23] F.K. Abdullaev, A. Gammal, L. Tomio and T. Frederico, Phys. Rev. A 63043604 (2001).

[24] H. Al-Jibbouri, I. Vidanovic, A. Balaz, and A. Pelster, J. Phys. B: At. Mol. Opt. Phys. 46, 065303 (2013).

[25] H-C Li, K-J. Chen and J-K Xue, Chin. Phys. Lett. 27 030304 (2010)

[26] Peng P and Li G-Q Chin. Phys. B 18, 3221 (2009).

[27] M. S. Mashayekhi, J.-S. Bernier, D. Borzov, J.-L. Song, and F. Zhou, Phys. Rev. Lett. 110, 145301 (2013).

[28] Zhen-Kai Lu, Yun Li, D. S. Petrov, and G. V. Shlyapnikov, Phys. Rev. Lett. 115, 075303 (2015).

[29] H. Kadau, M. Schmitt, M. Wenzel, C. Wink, T. Maier, I. Ferrier-Barbut and T. Pfau, Nature 530 ,194 (2016).

[30] Kui-Tian Xi and Hiroki Saito, Phys. Rev. A 93, 011604(R) (2016).

[31] P. B. Blakie, Phys. Rev. A 93, 033644 (2016).

[32] L. Chomaz, S. Baier, D. Petter, M. J. Mark, F. Wächtler, L. Santos and F. Ferlaino, Phys. Rev. X 6, 041039 (2016).

[33] A. Boudjemâa, Annals of Physics, 381, 68 (2017).

[34] A. Boudjemâa, J. Phys. B: At. Mol. Opt. Phys. 51, 2 (2017).

[35] E. Braaten and A. Nieto, Eur. Phys. J. B 11, 143 (1999).

[36] A. X. Zhangand and J. K. Xue, Phys.Rev. A 75, 013624 (2007).

[37] P. J. Everitt, M. A. Sooriyabandara, G. D. McDonald, K. S.Hardman, C. Quinlivan, M. Perumbil, P. Wigley, J. E. Debs, J. D. Close, C. C. N. Kuhn, N. P. Robins, arXiv:1509.06844v1 (2015).

[38] E. Braaten, H.-W. Hammer, and T. Mehen, Phys. Rev. Lett. 88, 040401 (2002). 
[39] K. Huang and H. F. Meng, Phys. Rev. Lett. 69, 644 (1992).

[40] A. Boudjemâa, J. Phys. B: At. Mol. Opt. Phys. 48035302 (2015).

[41] J. O. Andersen, Rev. Mod. Phys 76, 599 (2004).

[42] V. I. Yukalov, Phys. Part. Nucl. 42, 460 (2011).

[43] A. Boudjemâa, Degenerate Bose Gas at Finite Temperatures, LAP LAMBERT Academic Publishing (2017).
[44] Alexander L. Fetter and John Dirk Walecka, Theoretical Mechanics of Particles and Continua, Dover Publications (2003).

[45] T. D. Lee, K. Huang and C. N. Yang, Phys. Rev 106, 1135 (1957). 